\title{
Synthesis of Novel 5-Phenylselenenyl-2,4-Disubstituted Pyrimidine Analogs
}

\author{
ISMAIL, UPENDAR REDDY CH and THALARI GANGADHAR*
}

Department of Chemistry, Nizam College, Bhasheerbhagh, Hyderabad-500001, Andhra Pradesh, India

gangadhar.thalari@gmail.com

Received 6 May 2012 / Accepted 18 May 2012

\begin{abstract}
Phenylselenenyl-2,4-disubstituted pyrimidine analogs were prepared efficiently in four steps. Thiol group of 2-thiouracil was protected with various alkylating agents in presence of base furnishes 2-alkylthiouracils (2a-c), these on reaction with phenylselenenyl chloride in pyridine under anhydrous conditions yielded 5-phenylselenenyl-2-alkylthiouracils (3a-c). Chlorination of 5-phenyl-selenenyl-2alkylthiouracils with excess $\mathrm{POCl}_{3}$ under reflux furnishes 5-phenylselenenyl-4-chloro-2alkylthiopyrimidines (4a-c). Aromatic nucleophilic substitution reaction of 5-phenyl-selenenyl-4-chloro2-alkylthiopyrimidines with oxygen nucleophiles like sodium phenoxides furnished the target compounds (5a-o) in 60-75\% yield. All the synthesized compounds were evaluated for antimicrobial activities.
\end{abstract}

Keywords: Pyrimidine analogs, 2-Alkyllthiouracils, 5-Phenylselenenylpyrimidines, 2,4,5-Trisubstituted pyrimidines

\section{Introduction}

Selenium, an essential trace mineral ${ }^{1}$ is a vital component of the selenoproteins specifically glutathione peroxidase mainly required for normal health and reproduction ${ }^{2}$. Selenium was discovered in 1818 by Swedish chemist Berzelius. It was considered as a poison until it was identified as a micronutrient for bacteria, mammals and birds ${ }^{3}$. Selenium is essential for the efficient and effective operation of the immune system in both animals and humans ${ }^{4}$. Organoselenium compounds have substantially greater bioavailability than that of inorganic selenium $^{5}$. More importantly, organic selenium is usually found to be less toxic than inorganic forms ${ }^{6}$. Organoselenium compounds have been tested as antibacterial, antiviral, antifungal, antiparasitic, antihistamine and anticancer agents ${ }^{7}$. During the last few years, tremendous effort has been directed towards the synthesis of stable organoselenium compounds that could be used as antioxidants, enzyme modulators, antitumors, antivirals, antimicrobials, antihypertensive agents and cytokine inducers ${ }^{8}$. Though several organoselenium compounds are known, they are less explored for their antibacterial properties. Phenylselenenyl substituted pyrimidines were synthesized and evaluated for the inhibition of enzymes involved in pyrimidine metabolism ${ }^{9-17}$ such as dihydrouracil dehydrogenase (DHUDase), orotate phosphoribosyl transfarase (OPRTase), Thymidine phosphorylase (Thdpase) and uridine phosphorylase (Urdpase). Herein, we report a facile methodology for the synthesis of various 5-phenylselenenyl-4-phenoxy-2-alkylthiopyrimidines starting from 2-thiouracil. 


\section{Experimental}

2-Thiouracil was purchased from Hi-Media Laboratories Pvt. Ltd., Mumbai (India), methyl iodide, ethyl iodide, benzyl chloride, $\mathrm{POCl}_{3}$ and phenols were purchased from SISCO Research Laboratories Pvt. Ltd., Mumbai (India). All the solvents used were laboratory grade or were purified according to standard procedures. Melting points were determined by using a Thomas-Hoover melting point apparatus and were uncorrected. IR spectra in $\mathrm{KBr}$ disc were recorded on Perkin-Elmer-Spectrum-one FT IR spectrophotometer $\left(v_{\max }\right.$ in $\left.\mathrm{cm}^{-1}\right)$ and ${ }^{1} \mathrm{H}$ NMR spectra in DMSO- $d_{6}$ and/or $\mathrm{CDCl}_{3}$ on amx $400,400 \mathrm{MHz}$ spectrophotometer using TMS as internal standard (chemical shift in $\delta$ or ppm). Mass spectra were recorded on a JEOL SX 102 Mass spectrometer using Argon/Xenon (6kv, $10 \mathrm{~mA}$ ) as the FAB gas. Purity of the compounds was checked by TLC using silica gel ' $G$ ' plates obtained from Whatman Inc and a fluorescent indicator. 2-alkylthiouracils $2 a-c$ were prepared by following the literature method $^{18 a-d}$.

General procedure for the preparation of 5-phenylselenenyl-2-alkylthiouracil (3a-c) Phenylselenenyl chloride $(0.006 \mathrm{~mol})$ was dissolved in dry pyridine (30 mL) and then 2-alkylthiouracil $(0.006 \mathrm{~mol})$ was added. The reaction mixture was stirred at $60-65{ }^{0} \mathrm{C}$ for $24 \mathrm{~h}$ under nitrogen atmosphere (monitored by TLC). The reaction mixture was allowed to cool to room temperature and then concentrated under vacuum to remove pyridine. The residue was co-evaporated with benzene and EtOH $(20: 10 \mathrm{~mL})$. The residue was loaded on silica gel column and eluted with $\mathrm{CHCl}_{3}$ to remove diphenyldiselenide. The product was then obtained on elution with $\mathrm{CHCl}_{3}$ : $\mathrm{MeOH}(95: 5)$ and TLC pure fractions were pooled and concentrated.

\section{2-Methylthio-5-phenylselenenyl pyrimidin-4(3H)-one (3a)}

Anal. Calcd. for $\mathrm{C}_{11} \mathrm{H}_{10} \mathrm{~N}_{2} \mathrm{OSSe}$ C, 44.45; N, 9.42. Found: C, 44.47; N, 9.45; IR ( $\left.\mathrm{KBr}, \mathrm{cm}^{-1}\right)$ : $3200(\mathrm{NH}), 1630(\mathrm{C}=\mathrm{O}), 1595(\mathrm{C}=\mathrm{N}) ;{ }^{1} \mathrm{H}$ NMR $\left(400 \mathrm{MHz}, \mathrm{CDCl}_{3}, \delta / \mathrm{ppm}\right): 2.50(\mathrm{~s}, 3 \mathrm{H}$, $\left.\mathrm{SCH}_{3}\right), 7.50-7.30\left(\mathrm{~m}, 5 \mathrm{H}, \mathrm{C}_{6} \mathrm{H}_{5}\right), 7.65\left(\mathrm{~s}, 1 \mathrm{H}, \mathrm{C}_{6} \mathrm{H}\right), 12.2$ (s, $\left.1 \mathrm{H}, \mathrm{NH}\right) ;{ }^{13} \mathrm{C}$ NMR $(100 \mathrm{MHz}$, $\left.\mathrm{CDCl}_{3}, \delta / \mathrm{ppm}\right): 170,165,139,133$ (2C), 130, 128, 127 (3C), 15; MS (m/z, (relative abundance, \%)): $298\left(\mathrm{M}^{+}, 100 \%\right)$.

\section{2-Ethylthio-5-phenylselenenyl pyrimidin-4(3H)-one (3b)}

Anal. Calcd. for $\mathrm{C}_{12} \mathrm{H}_{12} \mathrm{~N}_{2}$ OSSe: C, 46.30; N, 9.00. Found: C, 44.32; N, 9.02; IR $\left(\mathrm{KBr}, \mathrm{cm}^{-1}\right)$ : $3190(\mathrm{NH}), 1620(\mathrm{C}=\mathrm{O}), 1585(\mathrm{C}=\mathrm{N}) ;{ }^{1} \mathrm{H}$ NMR $\left(400 \mathrm{MHz}, \mathrm{CDCl}_{3}, \delta / \mathrm{ppm}\right): 1.30(\mathrm{t}, 3 \mathrm{H}$, $\mathrm{SCH}_{2} \mathrm{CH}_{3}$ ), 3.20 (q, 2H, $\left.\mathrm{SCH}_{2} \mathrm{CH}_{3}\right), 7.55-7.35$ (m, 5H, $\left.\mathrm{C}_{6} \mathrm{H}_{5}\right), 7.68\left(\mathrm{~s}, 1 \mathrm{H}, \mathrm{C}_{6} \mathrm{H}\right), 12.5$ (s, $1 \mathrm{H}, \mathrm{NH}) ;{ }^{13} \mathrm{C}$ NMR (100 MHz, $\left.\mathrm{CDCl}_{3}, \delta / \mathrm{ppm}\right): 172,163,139,133$ (2C), 130, 128, 127 (3C), 26, 16; MS ( $\mathrm{m} / \mathrm{z}$, (relative abundance, \%)): $312\left(\mathrm{M}^{+}, 100 \%\right)$.

\section{2-Benzylthio-5-phenylselenenyl pyrimidin-4(3H)-one (3c)}

Anal. Calcd. for $\mathrm{C}_{17} \mathrm{H}_{14} \mathrm{~N}_{2}$ OSSe: C, 54.69: N, 7.50. Found: C, 54.67; N, 7.51; IR (KBr): $\mathrm{NH}$ 3180, $\mathrm{C}=\mathrm{O}$ 1636, $-\mathrm{C}=\mathrm{N}-1591 \mathrm{~cm}^{-1}$; ${ }^{1} \mathrm{H}$ NMR $\left(400 \mathrm{MHz}, \mathrm{CDCl}_{3}, \delta / \mathrm{ppm}\right): 4.36(\mathrm{~s}, 2 \mathrm{H}$, $\left.\mathrm{SCH}_{2} \mathrm{Ph}\right) ; 7.53-7.26$ (m, 10H, $\left.\mathrm{C}_{6} \mathrm{H}_{5}\right), 7.79$ (s, $\left.1 \mathrm{H}, \mathrm{C}_{6} \mathrm{H}\right), 12.4$ (s, $\left.1 \mathrm{H}, \mathrm{NH}\right) ;{ }^{13} \mathrm{C}$ NMR $(100$ $\mathrm{MHz}, \mathrm{CDCl}_{3}, \delta$ / ppm): 175, 162, 140, 136, 132 (2C), 130 (2C), 127 (5C), 125 (3C), 37; MS $\left(\mathrm{m} / \mathrm{z}\right.$, (relative abundance, \%)): $374\left(\mathrm{M}^{+}, 100 \%\right)$.

General procedure for the preparation of 5-phenylselenenyl-4-chloro-2-alkylthiopyrimidines $(\mathbf{4 a}-\mathbf{c})$

A mixture of 5-phenylselenenyl-2-alkylthio uracils (3a-c) $(0.01 \mathrm{~mol})$ and $\mathrm{POCl}_{3}(0.1$ mole) was refluxed for 3 hrs (monitored by TLC). Excess $\mathrm{POCl}_{3}$ was removed under reduced pressure and the mixture was treated with ice-cold water. The separated solid was extracted 
with ether and washed with aq. $\mathrm{NaHCO}_{3}(5 \%)$ solution. Ether layer was collected and dried over anhydrous sodium sulphate and after solvent evaporation yielded the title compounds 4a-c and were recrystallized from $\mathrm{EtOH}$.

\section{4-Chloro-2-methylthio-5-phenylselenenyl pyrimidine (4a)}

Anal. Calcd. for $\mathrm{C}_{11} \mathrm{H}_{9} \mathrm{ClN}_{2} \mathrm{SSe}$ : C, 41.85; N, 8.87. Found: C, 41.87; N, 8.89; ${ }^{1} \mathrm{H}$ NMR $\left(400 \mathrm{MHz}, \mathrm{CDCl}_{3}, \delta / \mathrm{ppm}\right): 2.53\left(\mathrm{~s}, 3 \mathrm{H}, \mathrm{SCH}_{3}\right), 7.25-7.40\left(\mathrm{~m}, 5 \mathrm{H}, \mathrm{C}_{6} \mathrm{H}_{5}\right), 7.70(\mathrm{~s}, 1 \mathrm{H}$, $\left.\mathrm{C}_{6} \mathrm{H}\right) ;{ }^{13} \mathrm{C}$ NMR (100 MHz, $\left.\mathrm{CDCl}_{3}, \delta / \mathrm{ppm}\right): 173,160,155,138$ (2C), 130, 127 (3C), 120, 15; MS ( $\mathrm{m} / \mathrm{z}$, (relative abundance, \%)): $316\left(\mathrm{M}^{+}, 100 \%\right)$.

\section{4-Chloro-2-ethylthio-5-phenylselenenyl pyrimidine (4b)}

Anal. Calcd. for $\mathrm{C}_{12} \mathrm{H}_{11} \mathrm{ClN}_{2} \mathrm{SSe}$ C, 43.71; N, 8.50. Found: C, 43.72; N, 8.51; ${ }^{1} \mathrm{H}$ NMR (400 MHz, $\mathrm{CDCl}_{3}, \delta / \mathrm{ppm}$ ): 1.27 (t, $3 \mathrm{H}, \mathrm{SCH}_{2} \mathrm{CH}_{3}$ ), 3.00 (q, 2H, $\mathrm{SCH}_{2} \mathrm{CH}_{3}$ ), 7.30-7.45 (m, 5H, $\mathrm{C}_{6} \mathrm{H}_{5}$ ), 7.86 (s, $\left.1 \mathrm{H}, \mathrm{C}_{6} \mathrm{H}\right) ;{ }^{13} \mathrm{C}$ NMR (100 MHz, $\left.\mathrm{CDCl}_{3}, \delta / \mathrm{ppm}\right): 172,160,156,137$ (2C), 131, 128 (3C), 121, 30, 16; MS ( $\mathrm{m} / \mathrm{z}$, (relative abundance, \%)): $330\left(\mathrm{M}^{+}, 100 \%\right)$.

\section{4-Chloro-2-benzylthio-5-phenylselenenyl pyrimidine (4c)}

Anal. Calcd. for $\mathrm{C}_{17} \mathrm{H}_{13} \mathrm{~N}_{2} \mathrm{SSeCl}$ C, 52.12; N, 7.15. Found: C, 52.14; N, 7.16; ${ }^{1} \mathrm{H}$ NMR (400 MHz, $\left.\mathrm{CDCl}_{3}, \delta / \mathrm{ppm}\right): 4.3\left(\mathrm{~s}, 2 \mathrm{H}, \mathrm{SCH}_{2} \mathrm{Ph}\right), 7.6-7.1\left(\mathrm{~m}, 10 \mathrm{H}, \mathrm{C}_{6} \mathrm{H}_{5}\right), 8.0\left(\mathrm{~s}, 1 \mathrm{H}, \mathrm{C}_{6} \mathrm{H}\right) ;{ }^{13} \mathrm{C}$ NMR $(100$ MHz, $\left.\mathrm{CDCl}_{3}, \delta / \mathrm{ppm}\right):$ 172, 160, 156, 137 (3C), 130, 128 (5C), 127 (3C), 120, 35; MS (m/z, (relative abundance, \%)): $392\left(\mathrm{M}^{+}, 100 \%\right)$.

General procedure for the preparation of 5-phenylselenenyl-4-phenoxy-2alkylthiopyrimidines (5a-o)

To a solution of appropriate phenol $(0.004$ mole $)$ in dry toluene $(10 \mathrm{~mL})$ was treated with $60 \% \mathrm{w} / \mathrm{v}$ sodium hydride $(0.004$ mole) in oil under an inert atmosphere. The mixture was warmed to $50-60{ }^{\circ} \mathrm{C}$ for $30 \mathrm{~min}$ to facilitate the formation of sodium salt. After all the sodium hydride had reacted, the suspension was cooled and a solution of 5-phenylselenenyl -4-chloro-2-alkylthiopyrimidines (4a-c) $(0.001 \mathrm{~mole})$ in toluene $(10 \mathrm{~mL})$ was added slowly at room temperature. After stirring the reaction mixture at $75-80{ }^{0} \mathrm{C}$ overnight (monitored by TLC), it was allowed to cool to room temperature and the mixture was treated with water (25 mL). The separated solid was extracted with ether $(3 \times 25 \mathrm{~mL})$ and washed with $10 \%$ aq sodium hydroxide $(3 \times 25 \mathrm{~mL})$. Ether layer was collected, dried over anhydrous sodium sulphate and evaporation of the solvent furnished the crude compounds, which were recrystallized from EtOH yielded the title compounds $\mathbf{5 a - o . ~}$

\section{2-Methylthio-4-phenoxy-5-phenylselenenyl pyrimidine (5a)}

Anal. Calcd. for $\mathrm{C}_{17} \mathrm{H}_{14} \mathrm{~N}_{2}$ OSSe: C, 54.69; N, 7.50. Found: C, 54.70; N, 7.52; ${ }^{1} \mathrm{H}$ NMR (400 $\mathrm{MHz}, \mathrm{CDCl}_{3}, \delta / \mathrm{ppm}$ ): 2.50 (s, $\left.3 \mathrm{H}, \mathrm{SCH}_{3}\right), 6.95-7.35\left(\mathrm{~m}, 10 \mathrm{H}, \mathrm{C}_{6} \mathrm{H}_{5}\right), 8.70\left(\mathrm{~s}, 1 \mathrm{H}, \mathrm{C}_{6} \mathrm{H}\right)$; ${ }^{13} \mathrm{C}$ NMR (100 MHz, $\left.\mathrm{CDCl}_{3}, \delta / \mathrm{ppm}\right): 180,170,160,155,138$ (2C), 130 (3C), 128 (3C), 125, 122 (2C), 106, 15; MS (m/z, (relative abundance, \%)): $374\left(\mathrm{M}^{+}, 100 \%\right)$.

\section{2-Methylthio-4-(o-tolyloxy)-5-phenylselenenyl pyrimidine (5b)}

Anal. Calcd. for $\mathrm{C}_{18} \mathrm{H}_{16} \mathrm{~N}_{2} \mathrm{OSSe}$ C, 55.81; H, 4.16; N, 7.23. Found: C, 55.83; H, 4.15; N, 7.25; ${ }^{1} \mathrm{H}$ NMR (400 MHz, $\mathrm{CDCl}_{3}, \delta / \mathrm{ppm}$ ): 2.15 (s, 3H, $\mathrm{CH}_{3}$ ), 2.51 (s, 3H, $\mathrm{SCH}_{3}$ ), 7.05-7.30 $\left(\mathrm{m}, 9 \mathrm{H}, \mathrm{C}_{6} \mathrm{H}_{5}\right), 8.65$ (s, $\left.1 \mathrm{H}, \mathrm{C}_{6} \mathrm{H}\right) ;{ }^{13} \mathrm{C} \mathrm{NMR}\left(100 \mathrm{MHz}, \mathrm{CDCl}_{3}, \delta / \mathrm{ppm}\right): 180,170,160,150$, 138 (2C), 135, 130, 128 (4C), 126, 124, 119, 106, 17, 15; MS (m/z, (relative abundance, \%)): $388\left(\mathrm{M}^{+}, 100 \%\right)$. 


\section{2-Methylthio-4-(m-tolyloxy)-5-phenylselenenyl pyrimidine (5c)}

Anal. Calcd. for $\mathrm{C}_{18} \mathrm{H}_{16} \mathrm{~N}_{2}$ OSSe: C, 55.81; N, 7.23. Found: C, 55.82; N, 7.24; ${ }^{1} \mathrm{H}$ NMR (400 $\mathrm{MHz} \mathrm{CDCl}_{3}, \delta / \mathrm{ppm}$ ): 2.13 (s, 3H, $\mathrm{CH}_{3}$ ), 2.49 (s, 3H, $\mathrm{SCH}_{3}$ ), 7.06-7.35 (m, 9H, $\mathrm{C}_{6} \mathrm{H}_{5}$ ), 8.62 (s, $\left.1 \mathrm{H}, \mathrm{C}_{6} \mathrm{H}\right)$; MS (m/z, (relative abundance, \%)): $388\left(\mathrm{M}^{+}, 100 \%\right)$.

\section{2-Methylthio-4-(p-tolyloxy)-5-phenylselenenyl pyrimidine (5d)}

Anal. Calcd. for $\mathrm{C}_{18} \mathrm{H}_{16} \mathrm{~N}_{2} \mathrm{OSSe}$ : C, 55.81; N, 7.23. Found: C, 55.80; N, 7.23; ${ }^{1} \mathrm{H}$ NMR (400 MHz, $\mathrm{CDCl}_{3}, \delta / \mathrm{ppm}$ ): 2.35 (s, 3H, $\mathrm{CH}_{3}$ ), 2.50 (s, 3H, $\mathrm{SCH}_{3}$ ), 7.06 (d, 2H, ArH), 7.09 (d, 2H, ArH), 7.307.45 (m, 5H, $\left.\mathrm{C}_{6} \mathrm{H}_{5}\right), 8.71$ (s, $\left.1 \mathrm{H}, \mathrm{C}_{6} \mathrm{H}\right)$; $\mathrm{MS}$ ( $\mathrm{m} / \mathrm{z}$, (relative abundance, \%)): 388 ( $\left.\mathrm{M}^{+}, 100 \%\right)$.

\section{2-Methylthio-4-(p-chlorophenoxy)-5-phenylselenenyl pyrimidine (5e)}

Anal. Calcd. for $\mathrm{C}_{17} \mathrm{H}_{13} \mathrm{ClN}_{2} \mathrm{OSSe}$ : C, 50.07; N, 6.87. Found: C, 50.08; N, 6.89; ${ }^{1} \mathrm{H}$ NMR (400 MHz, $\mathrm{CDCl}_{3}, \delta / \mathrm{ppm}$ ): 2.50 (s, 3H, $\mathrm{SCH}_{3}$ ), 7.08 (d, 2H, ArH), 7.12 (d, 2H, ArH), 7.30$7.48\left(\mathrm{~m}, 5 \mathrm{H}, \mathrm{C}_{6} \mathrm{H}_{5}\right), 8.70$ (s, $\left.1 \mathrm{H}, \mathrm{C}_{6} \mathrm{H}\right)$; $\mathrm{MS}\left(\mathrm{m} / \mathrm{z}\right.$, (relative abundance, \%)): $408\left(\mathrm{M}^{+}, 100 \%\right)$.

\section{2-Ethylthio-4-phenoxy-5-phenylselenenyl pyrimidine (5f)}

Anal. Calcd. for $\mathrm{C}_{18} \mathrm{H}_{16} \mathrm{~N}_{2}$ OSSe: C, 55.81; N, 7.23. Found: C, 55.82; N, 7.25; ${ }^{1} \mathrm{H}$ NMR (400 $\mathrm{MHz}, \mathrm{CDCl}_{3}, \delta / \mathrm{ppm}$ ): 1.30 (t, $\left.3 \mathrm{H}, \mathrm{CH}_{3}\right), 3.2\left(\mathrm{q}, 2 \mathrm{H}, \mathrm{SCH}_{2}\right.$ ), 6.95-7.45 (m, $\left.10 \mathrm{H}, \mathrm{C}_{6} \mathrm{H}_{5}\right), 8.68$ (s, $\left.1 \mathrm{H}, \mathrm{C}_{6} \mathrm{H}\right) ;{ }^{13} \mathrm{C}$ NMR (100 MHz, $\left.\mathrm{CDCl}_{3}, \delta / \mathrm{ppm}\right): 170,168,160,155,137$ (2C), 129 (3C), 128 (3C), 124, 122 (2C), 105, 30, 15; MS (m/z, (relative abundance, \%)): 388 (M+100\%).

\section{2-Ethylthio-4-(o-tolyloxy)-5-phenylselenenyl pyrimidine (5g)}

Anal. Calcd. for $\mathrm{C}_{19} \mathrm{H}_{18} \mathrm{~N}_{2} \mathrm{OSSe}$ : C, 56.85; N, 6.98. Found: C, 56.86; N, 6.99; ${ }^{1} \mathrm{H}$ NMR (400 $\mathrm{MHz}, \mathrm{CDCl}_{3}, \delta / \mathrm{ppm}$ ): 1.30 (t, 3H, $\mathrm{CH}_{3}$ ), 2.15 (s, 3H, $\mathrm{CH}_{3}$ ), 3.2 (q, $2 \mathrm{H}, \mathrm{SCH}_{2}$ ), 7.05-7.30 $\left(\mathrm{m}, 9 \mathrm{H}, \mathrm{C}_{6} \mathrm{H}_{5}\right), 8.65$ (s, $\left.1 \mathrm{H}, \mathrm{C}_{6} \mathrm{H}\right) ;{ }^{13} \mathrm{C}$ NMR $\left(100 \mathrm{MHz}, \mathrm{CDCl}_{3}, \delta / \mathrm{ppm}\right): 171,165,158$, 154, 135 (2C), 130 (3C), 129 (3C), 125, 121 (2C), 106, 32, 17, 15; MS (m/z, (relative abundance, \%)): $402\left(\mathrm{M}^{+}, 100 \%\right)$.

\section{2-Ethylthio-4-(m-tolyloxy)-5-phenylselenenyl pyrimidine (5h)}

Anal. Calcd. for $\mathrm{C}_{19} \mathrm{H}_{18} \mathrm{~N}_{2}$ OSSe: C, 56.85; N, 6.98. Found: C, 56.84; N, 6.97; ${ }^{1} \mathrm{H}$ NMR (400 $\left.\mathrm{MHz}, \mathrm{CDCl}_{3}, \delta / \mathrm{ppm}\right): 1.30$ (t, $\left.3 \mathrm{H}, \mathrm{CH}_{3}\right), 2.13$ (s, 3H, $\mathrm{CH}_{3}$ ), 3.2 (q, 2H, $\mathrm{SCH}_{2}$ ), 7.06-7.35 (m, 9H, $\left.\mathrm{C}_{6} \mathrm{H}_{5}\right), 8.62$ (s, $\left.1 \mathrm{H}, \mathrm{C}_{6} \mathrm{H}\right)$; $\mathrm{MS}\left(\mathrm{m} / \mathrm{z}\right.$, (relative abundance, \%)): $402\left(\mathrm{M}^{+}, 100 \%\right)$.

\section{2-Ethylthio-4-(p-tolyloxy)-5-phenylselenenyl pyrimidine (5i)}

Anal. Calcd. for $\mathrm{C}_{19} \mathrm{H}_{18} \mathrm{~N}_{2}$ OSSe: C, 56.85; N, 6.98. Found: C, 56.83; N, 6.97; ${ }^{1} \mathrm{H}$ NMR (400 $\left.\mathrm{MHz}, \mathrm{CDCl}_{3}, \delta / \mathrm{ppm}\right): 1.30\left(\mathrm{t}, 3 \mathrm{H}, \mathrm{CH}_{3}\right), 2.35\left(\mathrm{~s}, 3 \mathrm{H}, \mathrm{CH}_{3}\right), 3.2\left(\mathrm{q}, 2 \mathrm{H}, \mathrm{SCH}_{2}\right), 7.06(\mathrm{~d}, 2 \mathrm{H}$, ArH), 7.09 (d, $2 \mathrm{H}, \mathrm{ArH}), 7.30-7.45\left(\mathrm{~m}, 5 \mathrm{H}, \mathrm{C}_{6} \mathrm{H}_{5}\right), 8.71$ (s, $\left.1 \mathrm{H}, \mathrm{C}_{6} \mathrm{H}\right) ; \mathrm{MS}(\mathrm{m} / \mathrm{z}$, (relative abundance, \%)): $402\left(\mathrm{M}^{+}, 100 \%\right)$.

\section{2-Ethylthio-4-(p-chlorophenoxy)-5-phenylselenenyl pyrimidine (5j)}

Anal. Calcd. for $\mathrm{C}_{18} \mathrm{H}_{15} \mathrm{ClN}_{2} \mathrm{OSSe}$ : C, 51.25; N, 6.64. Found: C, 51.27; N, 6.65; ${ }^{1} \mathrm{H}$ NMR (400 MHz, $\mathrm{CDCl}_{3}, \delta / \mathrm{ppm}$ ): 1.30 (t, 3H, $\mathrm{CH}_{3}$ ), 3.2 (q, 2H, $\mathrm{SCH}_{2}$ ), 7.08 (d, 2H, ArH), 7.12 (d, $2 \mathrm{H}, \mathrm{ArH}), 7.30-7.48\left(\mathrm{~m}, 5 \mathrm{H}, \mathrm{C}_{6} \mathrm{H}_{5}\right), 8.70\left(\mathrm{~s}, 1 \mathrm{H}, \mathrm{C}_{6} \mathrm{H}\right)$; $\mathrm{MS}(\mathrm{m} / \mathrm{z}$, (relative abundance, $\%)$ ): $422\left(\mathrm{M}^{+}, 100 \%\right)$.

\section{2-Benzylthio-4-phenoxy-5-phenylselenenyl pyrimidine (5k)}

Anal. Calcd. for $\mathrm{C}_{23} \mathrm{H}_{18} \mathrm{~N}_{2}$ OSSe: C, 61.47; N, 6.23. Found: C, 61.50; N, 6.25; ${ }^{1} \mathrm{H}$ NMR (400 $\left.\mathrm{MHz}, \mathrm{CDCl}_{3}, \delta / \mathrm{ppm}\right): 4.4\left(\mathrm{~s}, 2 \mathrm{H}, \mathrm{SCH}_{2}\right), 6.95-7.45\left(\mathrm{~m}, 15 \mathrm{H}, \mathrm{C}_{6} \mathrm{H}_{5}\right), 8.68\left(\mathrm{~s}, 1 \mathrm{H}, \mathrm{C}_{6} \mathrm{H}\right) ;{ }^{13} \mathrm{C}$ NMR (100 MHz, CDCl $3, \delta /$ ppm): 170 (2C), 160, 155, 137 (3C), 129 (3C), 128 (5C), 127 (3C), 124, 122 (2C), 105, 35; MS (m/z, (relative abundance, \%)): $450\left(\mathrm{M}^{+}, 100 \%\right)$. 


\section{2-Benzylthio-4-(o-tolyloxy)-5-phenylselenenyl pyrimidine (5I)}

Anal. Calcd. for $\mathrm{C}_{24} \mathrm{H}_{20} \mathrm{~N}_{2} \mathrm{OSSe}$ : C, 62.20; N, 6.04. Found: C, 62.18; N, 6.02; ${ }^{1} \mathrm{H}$ NMR (400 $\mathrm{MHz}, \mathrm{CDCl}_{3}, \delta / \mathrm{ppm}$ ): 2.10 (s, 3H, $\left.\mathrm{CH}_{3}\right), 4.40$ (s, $2 \mathrm{H}, \mathrm{SCH}_{2}$ ), 6.97-7.47 (m, 15H, $\left.\mathrm{C}_{6} \mathrm{H}_{5}\right), 8.65$ (s, $\left.1 \mathrm{H}, \mathrm{C}_{6} \mathrm{H}\right) ;{ }^{13} \mathrm{C}$ NMR (100 MHz, $\left.\mathrm{CDCl}_{3}, \delta / \mathrm{ppm}\right): 172$ (2C), 161, 153, 138 (3C), 130 (3C), 125 (5C), 125 (3C), 121, 122 (2C), 103, 36, 16; MS ( $\mathrm{m} / \mathrm{z}$, (relative abundance, \%)): 464 ( $\left.\mathrm{M}^{+}, 100 \%\right)$.

\section{2-Benzylthio-4-(m-tolyloxy)-5-phenylselenenyl pyrimidine (5m)}

Anal. Calcd. for $\mathrm{C}_{24} \mathrm{H}_{20} \mathrm{~N}_{2}$ OSSe: C, 62.20; N, 6.04. Found: C, 62.21; N, 6.06; ${ }^{1} \mathrm{H}$ NMR (400 $\mathrm{MHz}, \mathrm{CDCl}_{3}, \delta / \mathrm{ppm}$ ): 2.13 (s, 3H, $\mathrm{CH}_{3}$ ), 4.41 (s, 2H, $\mathrm{SCH}_{2}$ ), 6.90-7.47 (m, 15H, $\mathrm{C}_{6} \mathrm{H}_{5}$ ), 8.65 (s, $\left.1 \mathrm{H}, \mathrm{C}_{6} \mathrm{H}\right)$; MS (m/z, (relative abundance, \%)): $464\left(\mathrm{M}^{+}, 100 \%\right)$.

\section{2-Benzylthio-4-(p-tolyloxy)-5-phenylselenenyl pyrimidine (5n)}

Anal. Calcd. for $\mathrm{C}_{24} \mathrm{H}_{20} \mathrm{~N}_{2}$ OSSe: C, 62.20; N, 6.04. Found: C, 62.20; N, 6.04; ${ }^{1} \mathrm{H}$ NMR (400 $\mathrm{MHz}, \mathrm{CDCl}_{3}, \delta / \mathrm{ppm}$ ): 2.17 (s, 3H, $\mathrm{CH}_{3}$ ), 4.45 (s, 2H, $\mathrm{SCH}_{2}$ ), 6.90-7.40 (m, 15H, $\mathrm{C}_{6} \mathrm{H}_{5}$ ), 8.60 (s, $\left.1 \mathrm{H}, \mathrm{C}_{6} \mathrm{H}\right)$; MS (m/z, (relative abundance, \%)): $464\left(\mathrm{M}^{+}, 100 \%\right)$.

\section{2-Benzylthio-4-(p-chlorophenoxy)-5-phenylselenenyl pyrimidine (5o)}

Anal. Calcd. for $\mathrm{C}_{23} \mathrm{H}_{17} \mathrm{ClN}_{2} \mathrm{OSSe}$ C, 57.09; N, 5.79. Found: C, 57.10; N, 5.80; ${ }^{1} \mathrm{H}$ NMR (400 MHz, $\left.\mathrm{CDCl}_{3}, \delta / \mathrm{ppm}\right): 4.38$ (s, 2H, $\left.\mathrm{SCH}_{2}\right), 7.30-7.45\left(\mathrm{~m}, 14 \mathrm{H}, \mathrm{C}_{6} \mathrm{H}_{5}\right), 8.67(\mathrm{~s}, 1 \mathrm{H}$, $\left.\mathrm{C}_{6} \mathrm{H}\right)$; MS ( $\mathrm{m} / \mathrm{z}$, (relative abundance, \%)): $484\left(\mathrm{M}^{+}, 100 \%\right)$.

\section{Results and Discussion}

2-Thiouracil 1 was reacted with alkylhalides in presence of base furnished 2-alkylthiouracils (2a-c) in 63-90\% yield (Scheme 1 and Table 1). The reaction of compounds 2a-c with phenylselenenyl chloride in dry pyridine under anhydrous condition at $60-65{ }^{\circ} \mathrm{C}$ for $24 \mathrm{~h}$ furnished the expected compound 5-phenylselenenyl-2-alkylthiouracils (3a-c) in 65-70\% yield. The IR spectrum of compound 3c shows characteristic absorptions of $\mathrm{NH}$ at 3180, C=O 1636 and $-\mathrm{C}=\mathrm{N} 1591 \mathrm{~cm}^{-1}$ respectively. Further, the structure of compound $3 \mathrm{c}$ was confirmed by the ${ }^{1} \mathrm{H}$ NMR spectrum and the characteristic signals are at $\delta 12.4(\mathrm{~s}, 1 \mathrm{H} . \mathrm{NH}), 7.79\left(\mathrm{~s}, 1 \mathrm{H}, \mathrm{C}_{6} \mathrm{H}\right)$, 7.53-7.26 (m, $\left.10 \mathrm{H}, \mathrm{C}_{6} \mathrm{H}_{5}\right), 4.36\left(\mathrm{~s}, 2 \mathrm{H}, \mathrm{SCH}_{2} \mathrm{Ph}\right)$. Further the structure of compound 3c was confirmed based on the mass spectral data, the molecular ion peak at $m / z=374\left(\mathrm{M}^{+1}, 100 \%\right)$. The ${ }^{1} \mathrm{H}$ NMR spectrum of the compound $3 \mathrm{c}$ indicates the phenylselenenyl group was attached to the $\mathrm{C}_{5}$-position of the pyrimidine ring. A significant deshielding effect of $\mathrm{C}_{6} \mathrm{H}$ was caused by the presence of a selenium substituent at the $\mathrm{C}_{5}$-position of the pyrimidine ring.

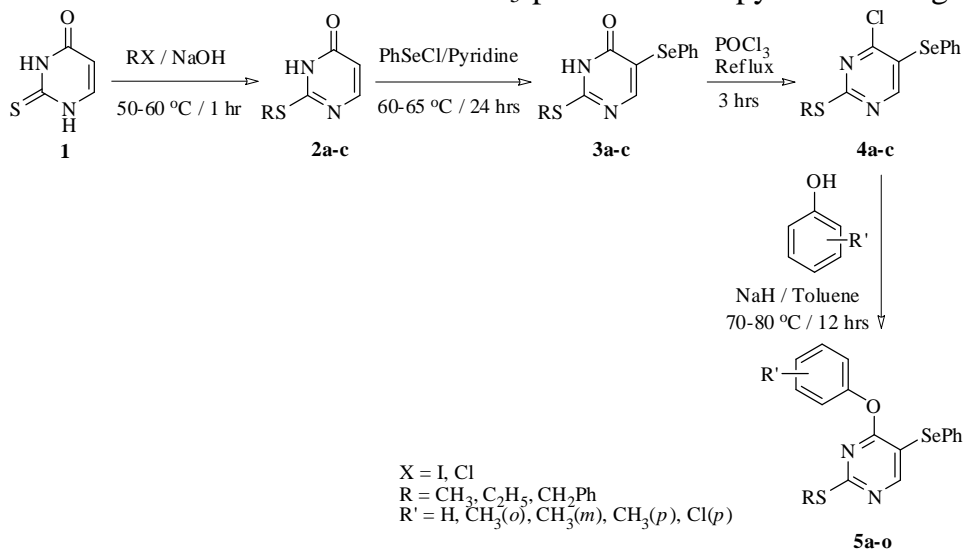

Scheme 1. Synthesis of 5-phenylselenenyl-2,4-disubstituted pyrimidines (5a-o) 
Table 1. Synthesis of 5-phenylselenenyl-4-substituted-2-benzylthiopyrimidines (5a-o)

\begin{tabular}{|c|c|c|c|c|}
\hline Compound & $\mathrm{R}$ & $\mathrm{R}^{\prime}$ & $\mathrm{mp}^{\mathrm{a}},{ }^{0} \mathrm{C}$ & Yield $^{\mathrm{b}}, \%$ \\
\hline $2 a^{18 a}$ and $18 c$ & $-\mathrm{CH}_{3}$ & - & 198 & 63 \\
\hline $2 b^{18 b}$ & $-\mathrm{CH}_{2} \mathrm{CH}_{3}$ & - & 59-61 & 70 \\
\hline $2 c^{18 d}$ & $-\mathrm{CH}_{2} \mathrm{Ph}$ & - & $174-175$ & 90 \\
\hline $3 a$ & $-\mathrm{CH}_{3}$ & - & $148-150$ & 68 \\
\hline $3 \mathbf{b}$ & $-\mathrm{CH}_{2} \mathrm{CH}_{3}$ & - & $161-163$ & 70 \\
\hline $3 c$ & $-\mathrm{CH}_{2} \mathrm{Ph}$ & - & $175-177$ & 65 \\
\hline $4 a$ & $-\mathrm{CH}_{3}$ & - & semi-solid & 80 \\
\hline $4 b$ & $-\mathrm{CH}_{2} \mathrm{CH}_{3}$ & - & semi-solid & 78 \\
\hline $4 c$ & $-\mathrm{CH}_{2} \mathrm{Ph}$ & - & $59-60$ & 90 \\
\hline $5 a$ & $-\mathrm{CH}_{3}$ & $-\mathrm{H}$ & semi-solid & 75 \\
\hline $5 b$ & $-\mathrm{CH}_{3}$ & $-\mathrm{CH}_{3}(\mathrm{o})$ & semi-solid & 70 \\
\hline $5 c$ & $-\mathrm{CH}_{3}$ & $-\mathrm{CH}_{3}(m)$ & semi-solid & 60 \\
\hline $5 d$ & $-\mathrm{CH}_{3}$ & $-\mathrm{CH}_{3}(p)$ & semi-solid & 72 \\
\hline $5 e$ & $-\mathrm{CH}_{3}$ & $-\mathrm{Cl}(p)$ & semi-solid & 70 \\
\hline $5 f$ & $-\mathrm{CH}_{2} \mathrm{CH}_{3}$ & $-\mathrm{H}$ & semi-solid & 62 \\
\hline $5 g$ & $-\mathrm{CH}_{2} \mathrm{CH}_{3}$ & $-\mathrm{CH}_{3}(\mathrm{o})$ & semi-solid & 64 \\
\hline $5 h$ & $-\mathrm{CH}_{2} \mathrm{CH}_{3}$ & $-\mathrm{CH}_{3}(m)$ & semi-solid & 62 \\
\hline $5 i$ & $-\mathrm{CH}_{2} \mathrm{CH}_{3}$ & $-\mathrm{CH}_{3}(p)$ & semi-solid & 60 \\
\hline $5 \mathbf{j}$ & $-\mathrm{CH}_{2} \mathrm{CH}_{3}$ & $-\mathrm{Cl}(p)$ & semi-solid & 70 \\
\hline $5 \mathbf{k}$ & $-\mathrm{CH}_{2} \mathrm{Ph}$ & $-\mathrm{H}$ & 67-69 & 70 \\
\hline 51 & $-\mathrm{CH}_{2} \mathrm{Ph}$ & $-\mathrm{CH}_{3}(\mathrm{o})$ & semi-solid & 72 \\
\hline $5 \mathrm{~m}$ & $-\mathrm{CH}_{2} \mathrm{Ph}$ & $-\mathrm{CH}_{3}(m)$ & semi-solid & 70 \\
\hline $5 n$ & $-\mathrm{CH}_{2} \mathrm{Ph}$ & $-\mathrm{CH}_{3}(p)$ & semi-solid & 65 \\
\hline 50 & $-\mathrm{CH}_{2} \mathrm{Ph}$ & $-\mathrm{Cl}(p)$ & semi-solid & 68 \\
\hline
\end{tabular}

${ }^{a}$ Melting points are uncorrected, ${ }^{b}$ Yield refers to purified product

In this case the reaction may proceed via the direct addition of phenylselenenyl chloride to the 5,6-double bond followed by elimination of $\mathrm{HCl}$ by pyridine. Compounds 3a-c on subjected to chlorination with $\mathrm{POCl}_{3}$ yielded 5-phenylselenenyl-4-chloro-2-alkylthiopyrimidines (4a-c) in $80-90 \%$ yield. The structural assignment of the compound $\mathbf{4 c}$ was based on the following spectral data, IR spectrum of compound $4 \mathbf{c}$ shows the absence of $\mathrm{NH}$ and $\mathrm{C}=\mathrm{O}$ absorptions at $3180,1636 \mathrm{~cm}^{-1}$ respectively, while it shows the presence of $\mathrm{C}-\mathrm{Cl}$ absorption at $735 \mathrm{~cm}^{-1}$. Further confirmation of the structure of compound $4 \mathrm{c}$ was based on the ${ }^{1} \mathrm{H}$ NMR spectrum; exhibiting the characteristic signals at $\delta 8.0\left(\mathrm{~s}, 1 \mathrm{H}, \mathrm{C}_{6} \mathrm{H}\right)$, 7.6-7.1 (m, $\left.10 \mathrm{H}, \mathrm{C}_{6} \mathrm{H}_{5}\right), 4.3\left(\mathrm{~s}, 2 \mathrm{H}, \mathrm{SCH}_{2} \mathrm{Ph}\right)$. Additional confirmation is the absence of $\mathrm{NH}$ peak at $\delta 12.4$ due to the aromatization of 5-phenylselenenyl-2-benzylthiouracil $3 \mathrm{c}$ to 5-phenylselenenyl-4-chloro-2-benzylthiopyrimidine 4c. Further the structure of compound 4c was confirmed based on the mass spectral data, the molecular ion peak at $\mathrm{m} / \mathrm{z}=392$ $\left(\mathrm{M}^{+1}, 100 \%\right)$. The title compounds 5 a-o were obtained by the reaction of compounds 4 a-c with oxygen nucleophiles such as sodium phenoxides in dry toluene under $\mathrm{N}_{2}$ atmosphere for overnight at room temperature, via the aromatic nucleophilic substitution reaction. Compound 5k was obtained in 70\% yield, having m.p. 67-69 ${ }^{\circ} \mathrm{C}$ as a brown crystalline solid. The ${ }^{1} \mathrm{H}$ NMR signals are at $\delta 8.22\left(\mathrm{~s}, 1 \mathrm{H}, \mathrm{C}_{6} \mathrm{H}\right), 7.65-7.35\left(\mathrm{~m}, 15 \mathrm{H}, \mathrm{C}_{6} \mathrm{H}_{5}\right), 4.35$ (s, $2 \mathrm{H}, \mathrm{SCH}_{2} \mathrm{Ph}$ ). Further the structure of compound $\mathbf{5 k}$ was confirmed based on the mass spectral data, the molecular ion peak at $\mathrm{m} / \mathrm{z}=450\left(\mathrm{M}^{+1}, 100 \%\right)$. 


\section{Conclusion}

In conclusion, we have developed novel and efficient synthesis of 2,4,5-trisubstituted pyrimidine analogs bearing four hetero atoms like N, O, S and Se.

\section{References}

1. Rayman M P, Proc Nutr Soc., 2002, 61, 203.

2. Rayman M P, Lancet., 2000, 356, 233.

3. Schwarz K and Foltz C M, J Am Chem Soc., 1957, 79, 3292.

4. $\quad$ Arthur J R, McKenzie R C and Beckett G J, J Nutr., 2003, 133, 1457 S.

5. Cantor A H, Langevin M L, Noguchi T and Scott M L, J Nutr., 1975, 105, 96.

6. (a) Arenholt-Bendsleve D, Abdulla M, Jepsrn A and Pedeson E, Trace Elem Med., 1988, 5, 29; (b) Khalil A M, Mutat Res., 1989, 224, 503-506; (c) Khalil A M, Toxicol Environ Chem., 1994, 41, 147.

7. Athayde-Filho P F, Souza A G, Morais S A, Botelho J R, Barbosa-Filho J M, Miller J and Lira B F, Arkivoc, 2004, vi, 22.

8. (a) Mugesh G, du Mont W W and Sies H, Chem Rev., 2001, 101, 2125; (b) SorianoGarcia M, Curr Med Chem., 2004, 11, 1657.

9. Bher J B, Gourlain T, Helimi A and Guillerm G, Bioorg Med Chem Lett., 2003, 13, 1713.

10. Niedzwicki J G, Kouni M H, Chu S H and Cha S, Biochem Pharmacol., 1981, 30, 2097.

11. Niedzwicki J G, Chu S H, Kouni M H, Rowe E C and Cha S, Biochem Pharmacol., 1982, 31, 1857.

12. Niedzwicki J G, Kouni M H, Chu S H and Cha S, Biochem Pharmacol., 1983, 32, 399.

13. Kouni M H, Naguib F N M, Chu S H, Cha S, Ueda T, Gosselin G, Imbach J L, Shealy Y F and Otter B A, Mol Pharmacol., 1988, 34, 104.

14. Naguib F N M, Kouni M H, Chu S H and Cha S, Biochem Pharmacol., 1987, 36, 2195.

15. Naguib F N M, Levesque D L, Wang E C, Panzica R P and Kouni M H, Biochem Pharmacol., 1993, 46, 1273.

16. Goudgaon N M, Naguib F N M, Kouni M H and Schinazi R F, J Med Chem., 1993, 36, 4250.

17. Goudgaon N M and Schinazi R F, J Med Chem., 1991, 34, 3305.

18. (a) Barrett H W, Goodman I and Dittmer K, J Amer Chem Soc., 948, 70, 1753;

(b) Wickliffe O W, Vanessa L S, Bhargavi V, Jeffrey A S, Linda M P and Jeehiun K L, Org Biomol Chem., 2008, 6, 4533; (c) Francesco T, Marco S, Kenneth M N, Giovanni P, Matteo P, Federico I, Stefano T and Andrea P, Tetrahedron, 2010, 66, 1280;

(d) David F, Montserrat H and Jose M V, J Combi Chem., 2003, 5(3), 311. 